\title{
FLEBOTOMÍNEOS (DIPTERA, PSYCHODIDAE) DE MATAS DE TERRA FIRME E DE VÁRZEA, DO MUNICÍPIO DE PARAGOMINAS, ESTADO DO PARÁ, BRASIL ${ }^{1}$
}

\author{
José Manuel Macário REBÊLO² ${ }^{2}$ Yrla Nívea OLIVEIRA-PEREIRA ${ }^{3}$
}

\begin{abstract}
RESUMO - Neste trabalho, a riqueza e a abundância das espécies de Lutzomyia foram estudadas nas matas secundárias de terra firme e de várzea, no municipio de Paragominas, Estado do Pará. Os flebotomíneos foram capturados das 18 às 6 horas em armadilhas luminosas CDC. Foram capturados 1.184 exemplares de 32 espécies, sendo 680 machos e 504 fêmeas. Na mata de terra firme foram encontradas 942 espécimens de 31 espécies, sendo as mais comuns L.complexa $(41,1 \%)$, L. corossoniensis $(11,6 \%)$, L. claustrei $(5,7 \%)$, L. infraspinosa $(5,7 \%)$, L. saulensis $(5,1 \%)$, L. flaviscutellata $(4,8 \%), L$. davisi $(3,5 \%)$ e $L$. wellcomei $(3,3 \%)$. As demais espécies representaram juntas $19,2 \%$. Na estação chuvosa a riqueza (24 espécies) e a abundância das espécies (38,8 espécimens/armadilha/noite) foram maiores do que na seca (16 espécies e 13,83 espécimens/armadilha/noite). Na mata de várzea foram capturados 242 espécimens de 12 espécies, o dominio foi de $L$. saulensis $(46,7 \%)$, L.complexa $(36 \%)$ e $L$. shawi $(7,4 \%)$. As demais espécies representaram juntas apenas $9,9 \%$. A riqueza de espécies (9) foi maior na estação chuvosa do que na seca (6), mas a abundância de espécimens foi maior no periodo seco (6,5 espécimens/ armadilha/noite) do que no chuvoso ( 5,85 espécimens/armadilha/noite).
\end{abstract}

Palavras-chave: Lutzomyia, Leishmaniose, Paragominas, Pará.

\section{Sand Flies (Diptera, Psychodidae) of the "Varzea" and "Terra Firme" Secondary Forests of the Municipal District of Paragominas, Pará State, Brazil'}

ABSTRACT - In this work, the richness and relative abundance of species of the genus Lutzomyia, in the "varzea" and "terra firme" secondary forests, in the municipal district of Paragominas, Pará State were studied. The sandflies were captured from 6:00 P.M. to 6:00 A.M. with CDC light-traps. A total of 1,184 specimens and 32 species were captured, being 680 males and 504 females. In the "terra firme" forest 942 specimens of 31 species were captured. L.complexa was the more frequent species, being represented by $41.1 \%$ of the captured specimens, followed, in order of importance by $L$. corossoniensis $(11.6 \%), L$. claustrei $(5.7 \%), L$. infraspinosa $(5.7 \%)$, L. saulensis $(5.1 \%)$, L. flaviscutellata $(4.8 \%)$, L. davisi $(3.5 \%)$ and $L$. wellcomei $(3,3 \%)$. The other species represented $19.2 \%$. In the rainy season the species richness (24) and abundance ( 38.8 specimens/light-trap/night) were higher than the dry season (16 species and 13.83 specimens/light-trap/night). In the "várzea" forest 242 specimens of 12 species were captured. The dominant species were L. saulensis $(46.7 \%)$, L.complexa $(36 \%)$ and L. shawi $(7.4 \%)$. The others species represented $9.9 \%$. The species richness (9) was higher in the rainy season than the dry season (6), but the specimen abundance was higher in the dry period (6.5 specimens/light-trap/night) than the rainy season ( 5.85 specimens/light-trap/night).

Key-words: Lutzomyia, Leishmaniasis, Paragominas, Pará.

${ }^{1}$ Trabalho financiado pela Biométrica Avaliações Ecológicas e Manejo Ambiental.

${ }^{2}$ Departamento de Biologia, Universidade Federal do Maranhão - Campus Universitário do Bacanga. Avenida dos Portugueses s/n 65.080-040 São Luís-MA, Brasil.

${ }^{3}$ Departamento de Biologia, Universidade Estadual do Maranhão, Campus Universitário Paulo VI - Tirirical - São Luis-MA, Brasil.

Correspondência: José Manuel Macário Rebêlo. Departamento de Patologia, Universidade Federal do Maranhão, Praça Madre Deus, ñ 02 65.025-560, São Luís-MA, Brasil. 


\section{INTRODUÇÃO}

No Estado do Pará, existem catalogadas aproximadamente 102 espécies de flebotomíneos, para as quais existe chave regional de identificação (Ryan, 1986). A distribuição de muitas delas, no Estado, já é relativamente bem conhecida (Damasceno et al. 1949; Martins et al. 1978), enquanto a de outras ainda carece de estudos.

Algumas espécies da fauna paraense também já foram bastante estudadas no que diz respeito aos aspectos bioecológicos e ao seu papel como vetores de leishmanias o que contribuiu muito para desvendar alguns aspectos da eco-epidemiologia das leishmanioses na Amazônia brasileira (Shaw \& Lainson, 1972; Fraiha et al. 1971, 1978; Lainson \& Shaw, 1987).

Ultimamente, os estudos associados à biologia e à ecologia dos flebotomineos paraenses têm sido concentrados, principalmente, nas espécies identificadas como vetores das várias formas de leishmanioses amazônicas (Lainson, 1981; Ready et al. 1983; Silveira et al. 1991; Souza et al. 1996).

Na Amazônia, as leishmanioses são zoonoses de mamiferos silvestres, e até o momento não existem evidências de transmissão domiciliar ou peridomiciliar da leishmaniose tegumentar americana (LTA), sendo, nesse caso, considerada uma doença ocupacional, acometendo principalmente madeireiros, lavradores, pescadores, topógrafos, biólogos e outros profissionais que entram nas matas para exercer suas atividades ou se deparam com os flebotomíneos.

Neste trabalho, apresenta-se a composição de espécies e a abundância relativa das populações de flebotomineos encontrados nos ambientes de matas de terra firme e de várzea, nas estações seca e chuvosa de áreas restritas do Estado do Pará.

O trabalho resultou de uma excursão realizada pelo Rio Capim, no município de Paragominas, com o intuito de estudar alguns grupos de insetos hematófagos de interesse para a saúde pública. Na ocasião, teve-se a oportunidade de capturar exemplares de várias espécies de flebotomíneos, as quais ainda não tinham registro de ocorrência nesse município, o que nos motivou a divulgar os dados aqui apresentados, que servirão para ampliar o conhecimento da distribuição desses importantes insetos no estado do Pará.

\section{MATERIAL E MÉTODOS}

O estudo consistiu em um levantamento de flebotomineos realizado no municipio de Paragominas, Estado do Pará nas matas secundárias de terra firme e de várzea, bastante alteradas pela atividade madeireira. Desenvolveu-se em duas etapas: a primeira, foi realizada na Fazenda Beira Rio, situada às margens do Rio Capim, na altura do $\mathrm{km} 150$ da jusante, de 21 a 24 de novembro de 1998 , na estação seca. Na segunda etapa, as coletas fo- 
ram feitas de 26 a 30 de abril de 1999 , na estação chuvosa, na altura do $\mathrm{km}$ 376 da jusante do rio, nos limites da Fazenda Maringá. Os pontos de coleta distavam no máximo $3 \mathrm{~km}$ da sede da Fazenda.

Os flebotomíneos foram capturados em armadilhas luminosas do tipo $\mathrm{CDC}$, das 18 às 6 horas. As armadilhas eram alimentadas por 4 pilhas alcalinas grandes de 1,5 volts e foram distribuidas nos ambientes de mata de várzea e de terra firme. Em cada ambiente, as armadilhas foram instaladas a $1,5 \mathrm{~m}$ de altura e funcionavam 12 horas/noite. Os insetos retidos nas armadilhas foram transferidos para câmaras mortíferas à base de acetato de etila, transferidos para frascos contendo álcool a $70 \% \mathrm{e}$ após isso foram transportados para o laboratório.

No laboratório, todos os espécimens foram transferidos para placas de Petri grandes, onde foram submetidos à triagem sob estereomicroscópio, separando-se os flebotomíneos dos outros insetos capturados, para posterior identificação, seguindo a proposta de Ryan (1986).

$\mathrm{Na}$ Fazenda Beira Rio os flebotomíneos foram capturados durante quatro noite consecutivas, sendo que no ambiente de terra firme foram utilizadas três armadilhas, totalizando 144 horas trabalhadas. Na várzea utilizaram-se duas armadilhas num total de 96 horas trabalhadas. Considerando os dois ambientes juntos foram 240 horas trabalhadas.
Na Fazenda Maringá as capturas ocorreram em cinco noites consecutivas e utilizaram-se quatro armadilhas em cada ambiente, resultando em 240 horas trabalhadas por ambiente e 480 horas no total.

\section{RESULTADOS}

Foram capturados 1.184 flebotomíneos de 32 espécies nas matas de terra firme e de várzea, sendo 680 machos e 504 fêmeas. Todas as espécies pertenciam ao gênero Lutzomyia, distribuidas nos subgêneros Evandromyia (2), Lutzomyia (1), Nyssomyia (8), Trichophoromyia (2), Psychodopygus (11), Pressatia (1), Viannamyia (1) e nos grupos Aragaoi (1), Braziliensis (1), Longispina (1), Oswaldoi (1), Shannoni (1), Saulensis (1) e Verrucarum (1), conforme lista:

\section{Subgênero Evandromyia}

L. brachyphala (Mangabeira, 1941)

L. infraspinosa (Mangabeira,1941)

\section{Subgênero Lutzomyia}

L. gomezi (Nitzulescu, 1930)

\section{Subgênero Nyssomyia}

L. antunesi (Coutinho, 1939)

L. anduzei (Rozeboom, 1942)

L. flaviscutellata (Mangabeira,1942)

L. umbratilis Ward \& Fraiha, 1977

L. olmecanociva Young \& Arias, 1982

L. shawi (Fraiha, Ward e Ready, 1981)

L. richardwardi (Ready e Fraiha, 1981)

L. whitmani (Antunes \& Coutinho, 1939)

\section{Subgênero Trichophoromyia}

L. ubiquitalis (Mangabeira, 1942) 
L. readyi Ryan, 1986

\section{Subgênero Pressatia}

Lutzomyia sp. (Mangabeira, 1942)

\section{Subgênero Psychodopygus}

L. amazonensis (Root, 1934)

L. ayrozai Barreto e Coutinho, 1940

L. chagasi (Costa Lima, 1947)

L. claustrei Abonnenc e Fauran, 1979

L. conossoniensis Le Pont e Pajot, 1978

L. carrerai carrerai (Barret, 1946)

L.complexa (Mangabeira, 1941)

L.davisi (Root, 1934)

L. hirsuta (Mangabeira, 1942)

L. paraensis (Forattini, 1973)

L.wellcomei Fraiha, Shaw e Lainson, 971

\section{Subgênero Viannamyia}

L. furcata (Mangabeira, 1941)

\section{Grupo Aragaoi}

L aragaoi (Costa Lima, 1932)

Grupo Braziliensis

L. lutziana (Costa Lima, 1932)

Grupo Longispina

L. dasypodogeton (Castro, 1939)

\section{Grupo Oswaldoi}

L. trinidadensis (Newstead, 1922)

\section{Grupo Shannoni}

L. shannoni (Dyar, 1929)

Grupo Saulensis

L. saulensis Floch e Abonnenc, 1944

Grupo Verrucarum

L. serrana Damasceno e Arouck, 1949

Mata de Terra Firme
Na mata de terra firme, foram encontradas 942 espécimens de 31 espécies (Tabela 1), sendo 59,8\% machos e $40,2 \%$ fềmeas. As espécies dominantes foram L.complexa $(41,1 \%)$, L. corossoniensis $(11,6 \%), L$. claustrei $(5,7 \%), L$. infraspinosa $(5,7 \%), L$. saulensis $(5,1 \%), L$. flaviscutellata $(4,8 \%)$, L. wellcomei $(3,3 \%)$ e $L$. davisi $(3,5 \%)$. As demais espécies representaram juntas $19,2 \%$.

$\mathrm{Na}$ estação chuvosa, foram encontradas 24 espécies e 776 espécimens, contra 16 espécies e 166 espécimens no periodo seco. Apesar das diferenças dos números de armadilhas utilizadas e de noites trabalhadas, entre as duas estações, detectou-se uma tendência dos flebotomíneos ocorrerem com maior abundância na estação chuvosa, tendo sido capturados 38,8 individuos/ armadilha/noite, contra 13,83 indivíduos capturados por armadilha/ noite na estação seca.

Durante as estações, houve substituição no domínio das espécies. $\mathrm{Na}$ estação seca as espécies mais comuns foram $L$. saulensis $(21,1 \%), L$. flaviscutellata $(18,1 \%), \quad L$. richardwardi $(13,9 \%)$, L infraspinosa $(13,9 \%), L$. wellcomei $(6,6 \%)$ e $L$. davisi $(6 \%)$, enquanto na estação chuvosa predominaram L.complexa $(49,4 \%), L$. corossoniensis (14\%), $L$. claustrei $(7,1 \%)$ e $L$. infraspinosa $(4 \%)$.

\section{Mata de Várzea}

$\mathrm{Na}$ mata de várzea, foram capturados 242 espécimens de 12 espécies (Tab. 2), havendo domínio 
das fêmeas $(51,7 \%)$ sobre os machos $(48,3 \%)$. As espécies mais abundantes foram $L$. saulensis $(46,7 \%)$, L.complexa $(36 \%)$ e $L$. shawi $(7,4 \%)$. As demais espécies representaram jun- tas apenas $9,9 \%$.

Do mesmo modo como ocorreu na mata de terra firme, na mata de várzea as espécies foram mais diversificadas na estação chuvosa,

Tabela 1. Espécies de flebotomineos capturados em mata de terra firme no municipio de Paragominas, Pará, nas estações seca/1998 e chuvosa/1999.

\begin{tabular}{|c|c|c|c|c|c|c|c|c|c|c|}
\hline \multirow{3}{*}{$\begin{array}{l}\frac{\text { Estações }}{\text { Espécies/Sexo }} \\
\text { L. anduzei }\end{array}$} & \multicolumn{4}{|c|}{ Seca (Fazenda Beira Rio) } & \multicolumn{5}{|c|}{ Chuvosa (Fazenda Maringá) } & \multirow{3}{*}{$\frac{1(\%)}{(1,1)}$} \\
\hline & \multirow[t]{2}{*}{ M } & \multirow[t]{2}{*}{$\mathrm{F}$} & \multicolumn{2}{|c|}{ Total (\%) } & \multirow{2}{*}{$\frac{M}{2}$} & \multirow{2}{*}{$\frac{F}{8}$} & \multicolumn{2}{|c|}{ Total $(\%)$} & \multirow{2}{*}{$\begin{array}{c}\text { Total } \\
10\end{array}$} & \\
\hline & & & & & & & 10 & $(1,3)$ & & \\
\hline L. antunesi & 3 & & 3 & $(1,8)$ & & 2 & 2 & $(0,2)$ & 5 & $(0,5)$ \\
\hline L. aragaoi & 1 & 3 & 4 & $(2,4)$ & & & & & 4 & $(0,4)$ \\
\hline L. ayrozai & & & & & 11 & 15 & 26 & $(3,4)$ & 26 & $(2,8)$ \\
\hline L. brachyphalla & & & & & 5 & 11 & 16 & $(2,0)$ & 16 & $(1,7)$ \\
\hline L. carrerai carrerai & & & & & 2 & 7 & 9 & $(1,2)$ & 9 & $(1,0)$ \\
\hline L. chagasi & & & & & 6 & & 6 & $(0,8)$ & 6 & $(0,6)$ \\
\hline L. claustrei & & & & & 16 & 38 & 54 & $(7,1)$ & 54 & $(5,7)$ \\
\hline L.complexa & 2 & 2 & 4 & $(2,4)$ & 359 & 24 & 383 & $(49,4)$ & 387 & $(41,1)$ \\
\hline L. corossoniensis & & & & & 1 & 108 & 109 & $(14,0)$ & 109 & $(11,6)$ \\
\hline L. dasypodogeton & 2 & & 2 & $(1,2)$ & & & & & 2 & $(0,2)$ \\
\hline L. davisi & 10 & & 10 & $(6,0)$ & 9 & 14 & 23 & $(3,0)$ & 33 & $(3,5)$ \\
\hline L. flaviscutellata & 20 & 10 & 30 & $(18,1)$ & 4 & 11 & 15 & $(1,9)$ & 45 & $(4,8)$ \\
\hline L. furcata & & 9 & 9 & $(5,4)$ & 4 & & 4 & $(0,4)$ & 13 & $(1,4)$ \\
\hline L. gomezi & & & & & & 1 & 1 & $(0,1)$ & 1 & $(0,1)$ \\
\hline L. hirsuta & & & & & 2 & 6 & 8 & $(1,0)$ & 8 & $(0,8)$ \\
\hline L. infraspinosa & 21 & 2 & 23 & $(13,9)$ & 24 & 7 & 31 & $(4,0)$ & 54 & $(5,7)$ \\
\hline L. Iutziana & & & & & 1 & & 1 & $(0,1)$ & 1 & $(0,1)$ \\
\hline L. paraensis & & & & & 4 & 2 & 6 & $(0,8)$ & 6 & $(0,6)$ \\
\hline L. (Pressatia) sp. & 1 & & 1 & $(0,6)$ & & & & & 2 & $(0,2)$ \\
\hline L. readyi & 1 & 3 & 4 & $(2,4)$ & & & & & 4 & $(0,4)$ \\
\hline L. richardwardi & 21 & 2 & 23 & $(13,9)$ & & & & & 23 & $(2,4)$ \\
\hline L. saulensis & 2 & 33 & 35 & $(21,1)$ & 1 & 12 & 13 & $(1,7)$ & 48 & $(5,1)$ \\
\hline L. serrana & & 3 & 3 & $(1,8)$ & & & & & 3 & $(0,3)$ \\
\hline L. shannoni & 1 & 1 & 2 & $(1,2)$ & & 1 & 1 & $(0,1)$ & 3 & $(0,3)$ \\
\hline L. shawi & & & & & 20 & 4 & 24 & $(3,2)$ & 24 & $(2,5)$ \\
\hline L. trinidadensis & & & & & & 2 & 2 & $(0,2)$ & 2 & $(0,2)$ \\
\hline L. ubiquitalis & & & & & & 10 & 10 & $(1,3)$ & 10 & $(1,1)$ \\
\hline L. umbratilis & & & & & 2 & & 2 & $(0,2)$ & 2 & $(0,2)$ \\
\hline L. wellcomei & 2 & 9 & 11 & $(6,6)$ & 2 & 18 & 20 & $(2,6)$ & 31 & $(3,3)$ \\
\hline L. whitmani & 1 & 1 & 2 & $(1,2)$ & & & & & 2 & $(0,2)$ \\
\hline Total & 88 & 78 & 166 & $(100,0)$ & 475 & 301 & 776 & $(100,0)$ & 9421 & $(100,0)$ \\
\hline$\%$ & 53 & 47 & 17 & 6 & 61,2 & 38,8 & & 2,4 & & \\
\hline
\end{tabular}


quando foram encontradas 9 espécies contra 6 na estação seca. Porém, a abundância de espécimens foi ligeiramente maior no período seco (6,5 espécimens/armadilha/noite) quando comparado com o periodo chuvoso (5,85 espécimens/armadilha/ noite),

Houve inversão de domínio entre os sexos e as estações. No periodo seco, a dominância foi das fêmeas ( $73,6 \%)$, já na estação chuvosa foram os machos que dominaram $(71,8 \%)$.

No periodo seco, a espécie mais freqüente foi $L$. saulensis, representando $90,4 \%$ dos exemplares capturados. As outras espécies representaram juntas $9,6 \%$ apenas. Já no periodo chuvoso quem dominou foi L. complexa $(74,4 \%)$ e $L$. shawi $(15,4 \%)$, tendo as demais espécies representado juntas $10,2 \%$ dos exemplares capturados.

Vale ressaltar que algumas espécies apresentaram grande densidade só de um sexo no ambiente de mata de terra firme, como
L.complexa, com 359 machos e $L$. corossoniensis, com 108 fêmeas. Já das espécies $L$. gomezi, L. shannoni, $L$. lutziana, L. whitmani e L. olmeca nociva só foi capturado um exemplar de cada, e as duas últimas só foram encontradas no ambiente de mata de várzea.

Nas noites da estação chuvosa em que as armadilhas foram colocadas na mata de várzea houve periodos de chuvas durante as capturas, o que provavelmente influenciou na densidade dos insetos neste ambiente.

\section{DISCUSSÃO}

Na região de Paragominas, onde foi realizado este estudo já foram encontradas cerca de 30 espécies (Ryan, 1986), mas neste levantamento capturaram-se 12 espécies que ainda não haviam sido registradas nesse municipio ( $L$. flaviscutellata, $L$. aragao $i, L$. chagasi, $L$ readyi, $L$. shawi, L. corossoniensis, L. anduzei, L. claustrei, L. lutziana, L. umbratilis,

Tabela 2. Espécies de flebotomineos capturados em mata de várzea, no municipio de Paragominas, Pará, nas estações seca/1998 e chuvosa/1999.

\begin{tabular}{|c|c|c|c|c|c|c|c|c|}
\hline Estações & Seca & zenda & Beira Rio) & Chuy & a (Faze & enda Maring & & \\
\hline Espécies/Sexo & $\mathrm{M}$ & $\mathrm{F}$ & Total (\%) & $\mathrm{M}$ & F & Total (\%) & Total & $(\%)$ \\
\hline L. anduzei & & & & & 3 & $3(2,7)$ & 3 & $(1,2)$ \\
\hline L.complexa & & & & 63 & 24 & $87(74,4)$ & 87 & $(36,0)$ \\
\hline L. davisi & 2 & & $2 \quad(1,6)$ & 3 & & $3(2,7)$ & 5 & $(2,1)$ \\
\hline L. flaviscutellata & 1 & & $1 \quad(0,8)$ & 1 & & $1(0,8)$ & 2 & $(0,8)$ \\
\hline L. furcata & 2 & 2 & $4 \quad(3,2)$ & & & & 4 & $(1,7)$ \\
\hline L. infraspinosa & 2 & & $2(1,6)$ & & & & 2 & $(0,8)$ \\
\hline L. olmeca nociva & & & & & 1 & I $\quad(0,8)$ & 1 & $(0,4)$ \\
\hline L. paraensis & & & & 1 & & $1 \quad(0,8)$ & 1 & $(0,4)$ \\
\hline L. saulensis & 26 & 87 & $113(90,4)$ & & & & 113 & $(46,7)$ \\
\hline L. shawi & & & & 15 & 3 & $18(15,4)$ & 18 & $(7,4)$ \\
\hline L. umbratilis & & & & & 2 & $2(1,6)$ & 2 & $(0,8)$ \\
\hline L. whitmani & & 3 & $3 \quad(2,4)$ & 1 & & I $(0,8)$ & 4 & $(1,7)$ \\
\hline Total & 33 & 92 & $125(100)$ & 84 & 33 & $117(100)$ & 242 & $(100)$ \\
\hline$\%$ & 26,4 & 73,6 & 52 & 71,8 & 28,2 & 48 & 100 & \\
\hline
\end{tabular}


L. trinidadensis e L. olmeca nociva), totalizando agora 42 espécies.

Houve uma diferença marcante na riqueza e densidade das espécies entre os dois tipos de matas, sendo maior na mata de terra firme do que na mata de várzea. Esses aspectos foram favorecidos também na estaçâo chuvosa, independente do tipo de mata. A baixa riqueza de espécies encontradas na várzea, quando comparada com a mata de terra firme pode ter sido ocasionada, em parte, pelas coletas coincidirem com noites de chuvas pesadas.

Considerando a diversidade e densidade das espécies dos subgêneros Psychodopygus e Nyssomyia, que contêm um grande número de representantes vetores, a estação chuvosa seria a mais favorável para a ocorrência desses flebotomineos e, talvez, a de maior risco para as pessoas adquirirem infecções leishmanióticas nas matas de Paragominas. Esses dados devem servir de alerta para o aprimoramento dos serviços de vigilância em saúde, no sentido de orientar as pessoas que entram em contato com as matas aqui estudadas.

Dentro desse contexto, destacam-se, nas florestas de Paragominas, as espécies L.complexa, $L$. wellcomei, $L$. flaviscutellata, $L$. umbratilis, L. ubiquitalis, L. whitmani e $L$. paraensis, por estarem diretamente relacionadas com o ciclo epidemiológico das leishmanioses.

As espécies L.complexa e $L$. wellcomei são vetoras de Leishmania braziliensis no estado do Pará. Neste estudo, L.complexa foi encontrada em baixa densidade na estação seca, porém muito comum na estação chuvosa, predominando sobre as demais espécies. Na Serra de Carajás, L. wellcomei ocorre nas florestas mais altas e pica o homem tanto durante o dia quanto à noite. A L.complexa predomina nas florestas mais baixas, podendo ser encontrada em outras áreas, menos onde a altitude aumenta (Fraiha et al. 1971). Do Amazonas em direção ao Maranhão, machos de $L$. wellcomei são capturados raramente (Ready et al. 1983), o que pôde ser comprovado neste levantamento, no qual o número de fềmeas foi superior cinco vezes ao de machos. A notável antropofilia e os hábitos diurnos das fêmeas de $L$. wellcomei reforçam a sua importância na transmissão das leishmanioses, visto que na maioria dos casos da doença, as pessoas só tiveram contato com a mata durante o dia, uma vez que esse mosquito não apresenta hábitos domiciliares (Ward et al. 1973, Castellón et al. 1994).

A espécie $L$, flaviscutellata é o agente vetor da $L$. amazonensis, causador da maioria dos casos de leishmaniose cutânea difusa (LCD), uma forma que não responde ao tratamento com as drogas usuais (Costa et al. 1992). É uma espécie estritamente silvestre e típica de áreas de várzeas, embora possa vir a adaptar-se às áreas de vegetação secundária. Devido à pouca atratividade que o homem exerce sobre as fêmeas dessa espécie, a infecção leishmaniótica está restrita às 
pessoas que penetram na floresta à noite (Lainson \& Shaw, 1979), principalmente na estação seca. Apresenta vôo baixo, tanto que no Estado do Amazonas foi capturada somente em armadilhas luminosas colocadas a $1 \mathrm{~m}$ de altura (Castellón et al. 1994).

L. umbratilis é reconhecidamente o agente vetor primário da $L$. guyanensis na Bacia Amazônica, onde causa a leishmaniose cutânea. Neste estudo foi encontrada apenas na estação chuvosa. De acordo com as observações de vários autores (Arias \& Freitas, 1978; Ready et al. 1983), essa espécie mantém o ciclo de transmissão silvestre durante a noite quando realiza a hematofagia, nas copas das árvores, em preguiça (Choloepus didactylus), tamanduá (Tamandua tetradactyla), mucura (Didelphis) e outros animais. Repousa na base dos troncos durante o dia, podendo transmitir a infecção para o homem, quando este penetra na floresta.

L. ubiquitalis foi capturada apenas na estação chuvosa e somente exemplares fêmeas. É a única espécie encontrada até o momento infectada com $L$. lainsoni. Já foi registrada na Serra de Carajás e em áreas de ocorrência de LTA, como Tucuruí. Raramente pica o homem, mas pode transmitir-lhe a infecção (Silveira et al. 1991).

$L$. whitmani é agente vetor da $L e$. shawi na Amazônia brasileira e da $L$. braziliensis nas outras áreas (Ready et al. 1997 e 1998). Nas áreas amazônicas, é essencialmente silvestre, sendo encontrado nas copas e troncos das árvores nas florestas. Em áreas não amazônicas é comum nos ambientes peri e intradomiciliares, mas na Amazônia maranhense essa espécie pode ser encontrada tanto no ambiente silvestre quanto nas habitações humanas das áreas rurais (Rebêlo et al. 1999). Vários estudos realizados no Brasil têm demonstrado que $L$. whitmani pode funcionar como vetora de mais que uma espécie de Leishmania, sendo vários os mamíferos considerados seus possíveis reservatórios, como primatas (Cebus apella), morcego (Chiropotes satanus), preguiça (Choloepus didactylus, Bradypus tridactylus) e quati (Nasua nasua). Conforme observado neste trabalho, sua densidade é maior nos meses úmidos do que nos meses secos (Lainson, 1981).

As espécies $L$. paraensis e $L$. ayrozai são vetoras da Le. naiffi. Neste estudo predominaram no ambiente de mata de terra firme e na estação chuvosa. Os parasitos já foram isolados em desdentados (tatu) no Pará, e alguns casos humanos de leishmaniose cutânea foram registrados no Amazonas.

L. furcata, L. richardwardi, $L$. corossoniensis e L. lutziana predominaram no ambiente de mata de terra firme, estando amplamente distribuídos na Amazônia. L. corossoniensis não ocorreu na estação seca, mas no periodo chuvosa ocorreu em alta densidade, especialmente as fềmeas.

\section{Bibliografia citada}

Arias, J.R; Freitas, RA. 1978. Sobre os vetores de leishmaniose cutânea na Amazônia Central do Brasil. 2. Incidência de flagelados em flebótomos selváticos. Acta 
Amazônica, 8: 387-396.

Castellón, E.G.; Arias, J.R.; Freitas, R.A.; Naiff, R.D. 1994. Os flebotomíneos da região amazônica, estrada ManausHumaitá, estado do Amazonas, Brasil (Diptera: Psychodidae: Phlebotominae). Acta Amazônica, 24: 91- 102.

Costa, J. M. L.; Saldanha, A. C. R.; MelloSilva, A. C.; Serra-Neto, A.; Galvão, C.E.S.; Pedroso-Silva, C.M.; Silva, A.R. 1992. Estado atual da leishmaniose cutânea difusa (LCD) no Estado do Maranhão. II. Aspectos epidemiológicos, clínicos e evolutivos. Rev. Soc. Bras. Med, Trop., 25: 115-123.

Damasceno, R.G.; Arouck, R.; Causey, O.R. 1949. Estudos sobre flebótomos no Vale Amazônico, parte VI. Contribuição ao conhecimento da distribuição geográfica e da incidência por tipo de captura, de 64 espécies identificadás. Rev. Serv. Esp. Saúde Pública, 2:817-842.

Fraiha, H.; Shaw, J.J,; Lainson, R. 1971. Phlebotominae brasileiros - II. Psychodopygus wellcomei, nova espécie antropófila de flebótomo do grupo squamiventris, do sul do estado do Pará, Brasil (Diptera, Psychodidae). Mem. Inst. Oswaldo Cruz, 69: 489-500.

Fraiha, H.; Ward, R.D.; Shaw, J.J; Lainson, R. 1978. Fauna antropófila de flebótomos da rodovia Transamazônica, Brasil (Diptera: Psychodidae), Bull. Pan. Amer. Health Org., 84:134-139.

Lainson, R. 1981. Epidemiologia e ecologia da leishmaniose tegumentar na Amazônia. Hiléia Médica, 3: 35-40.

Lainson, R.; Shaw, J.J. 1979. The role of animals in the epidemiology of South American leishmaniasis. In: Lumsden, Evans DA (ed) Biology of the Kinetoplastida, Academic Press, London, v. 2, p.1-116.

Lainson, R.; Shaw, J.J. 1987. Evolution, classification and geographical distribution. In: Peters, W.., Killiek Kendrick, R. (Ed.). The Leishmaniasis in biology and medicine. London: Academic Press, v.1, p. 1120.

Martins, A.V.; Williams, P.; Falcão, A. L. 1978.
American sanflies. Rio de Janeiro: Academia Brasileira de Ciências, 195p.

Ready, P.D.; Ribeiro, A.L.; Lainson, R.; Alencar, J.E;; Shaw, J.J. 1983. Presence of Psychodopygus wellcomei (Diptera: Psychodidae) a proved vector of the Leishmania braziliensis braziliensis in Ceará State. Mem. Inst. Oswaldo Cruz, 78: 235-236.

Ready, P.D.; Day, J.C.; Souza, A.A.A.; Rangel, E.F.; Davies, C.R. 1997. Mitochondrial DNA characterization of populations of Lutzomyia whitmani (Diptera, Psychodidae) incriminated in the peridomestic and silvatic transmission of Leishmania species in Brazil. Bul. Entomol. Research, 87: 187-195.

Ready, P.D.; Souza, A.A.A.; Rebêlo, J.M.M.; Day, J.C.; Silveira, F.; CampbellLendrum, D.; Davies, C.R.; Costa, J.M.L. 1998. Phylogenetic species and domesticity of Lutzomyia whitmani at the southeast boundary of Amazonian Brazil. Trans. R. Soc. Trop. Med. Hyg, 92: 159160.

Rebêlo, J.M.M.; Leonardo, E.S.; Costa, J.M.L.; Pereira, Y.N.O; Silva, F.S. 1999. Flebotomíneos (Diptera, Psychodidae) de área endêmica de leishmaniose na região dos cerrados, Estado do Maranhão, Brasil. Cad. Saú. Púb., 15: 623-630.

Ryan, L. 1986. Flebótomos do Estado do Pará, Brasil. Documento Técnico do Instituto Evandro Chagas, Belém, Pará, Brasil.

Shaw, J.J.; Lainson, R. 1972. Leishmaniasis in Brazil IV. Observations on the seasonal variation of Lutzomyia flaviscutellata in different types of forest and its relationship to enzootic rodent leishmaniasis (Leishmania mexicana amazonensis). Trans, R. Soc. Trop. Med. Hyg., 66: 709-717.

Silveira, F.T.; Souza, A.A.A.; Lainson, R.; Shaw, J.J; Braga, R.R.; Ishikawa, E.A.Y. 1991. Cutaneous leishmaniasis in the Amazon region: natural infection of the sandfly Lutzomyia ubiquitalis (Psychodidae: Phlebotominae) by Leishmania (Viannia) lainsoni in Pará State, Brazil. Mem, Inst. Oswaldo Cruz, 86: 127-130. 
Souza, A.A.A.; Ishikawa, E.; Braga, R.; Silveira, F; Lainson, R.; Shaw, J.J. 1996. Psychodopygus complexus, a new vector of Leishmania braziliensis to humans in Parà State, Brazil. Trans. R. Soc. Trop. Med. Hyg., 90: 112-113.

Ward, R.D.; Shaw, J.J.; Lainson, R. 1973. Leishmaniasis in Brazil. VIII. Observations on the phlebotominae fauna of an area highly endemic for cutaneous leishmaniasis in the Serra dos Carajás, Pará State, Trans. R. Soc. Trop. Med. Hyg., 67: 174-183. 